\title{
Distribution of NWG/RFCs Through the NIC
}

Status of this Memo

This memo provides information for the Internet community. This memo does not specify an Internet standard of any kind. Distribution of this memo is unlimited.

Notice

The Network Information Center (NIC), administered by Doug Engelbart at SRI, has established (or is establishing) lines of communication with all of the sites. The mechanism is for each site to have a Station Agent and a Technical Liaison Contact. The Station Agent maintains a small library of documents which arrive from the NIC. The Technical Liaison Contact matches requests from the network community to people or services at his site, and vice versa.

In my opinion, the current mailing list for the Network Working Group seems to duplicate in large part the NIC's distribution system. Therefore, I am making the following changes.

(1) Each site is presumed to have a Technical Liaison Contact. Where none has been appointed, the current receiver of NWG notes for that site will be assumed to be the Technical Liaison Contact. (Each site may change its Technical Liaison Contact by notifying the NIC.)

(2) NWG notes will be sent only to Technical Liaison Contacts and to those on the current mailing list who are not at a site.

(3) RFC numbers and NIC numbers will continue to be assigned by Jeanne North at SRI -- (415) 326-6200, ext. 4119, or use Enterprise numbers:

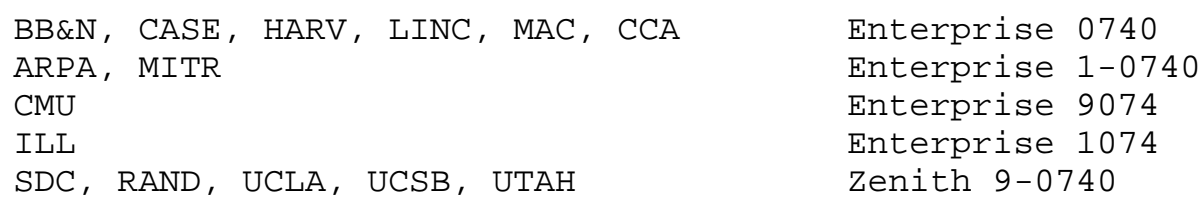


(4) NWG notes may be sent to the NIC for distribution, or may be distributed directly. If an author intends to distribute an NWG note himself, he should so inform Mrs. North and obtain any changes to the mailing list. All mailing should be airmail or first class, depending upon distance.

Those on the current mailing list who are not at sites are

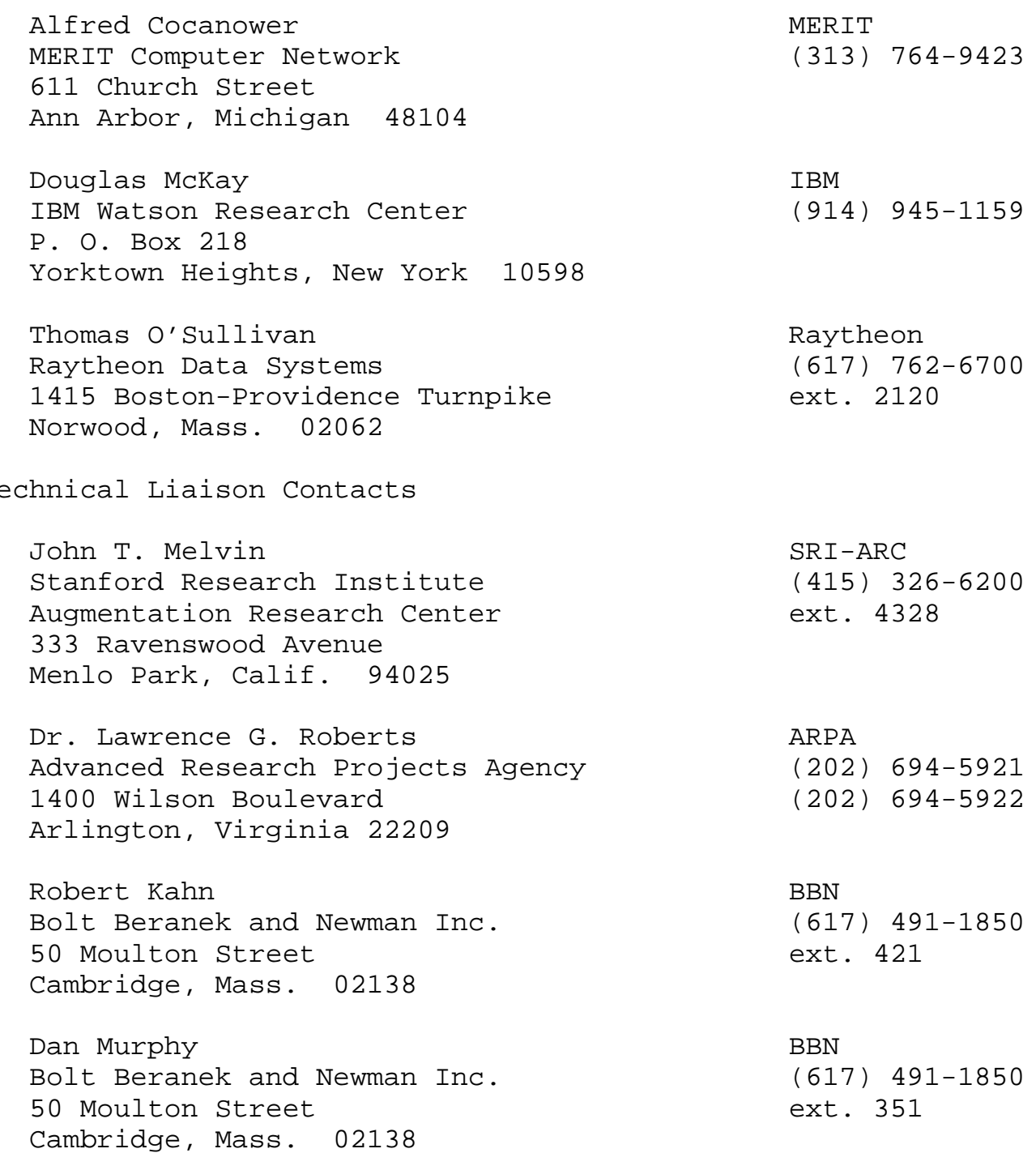




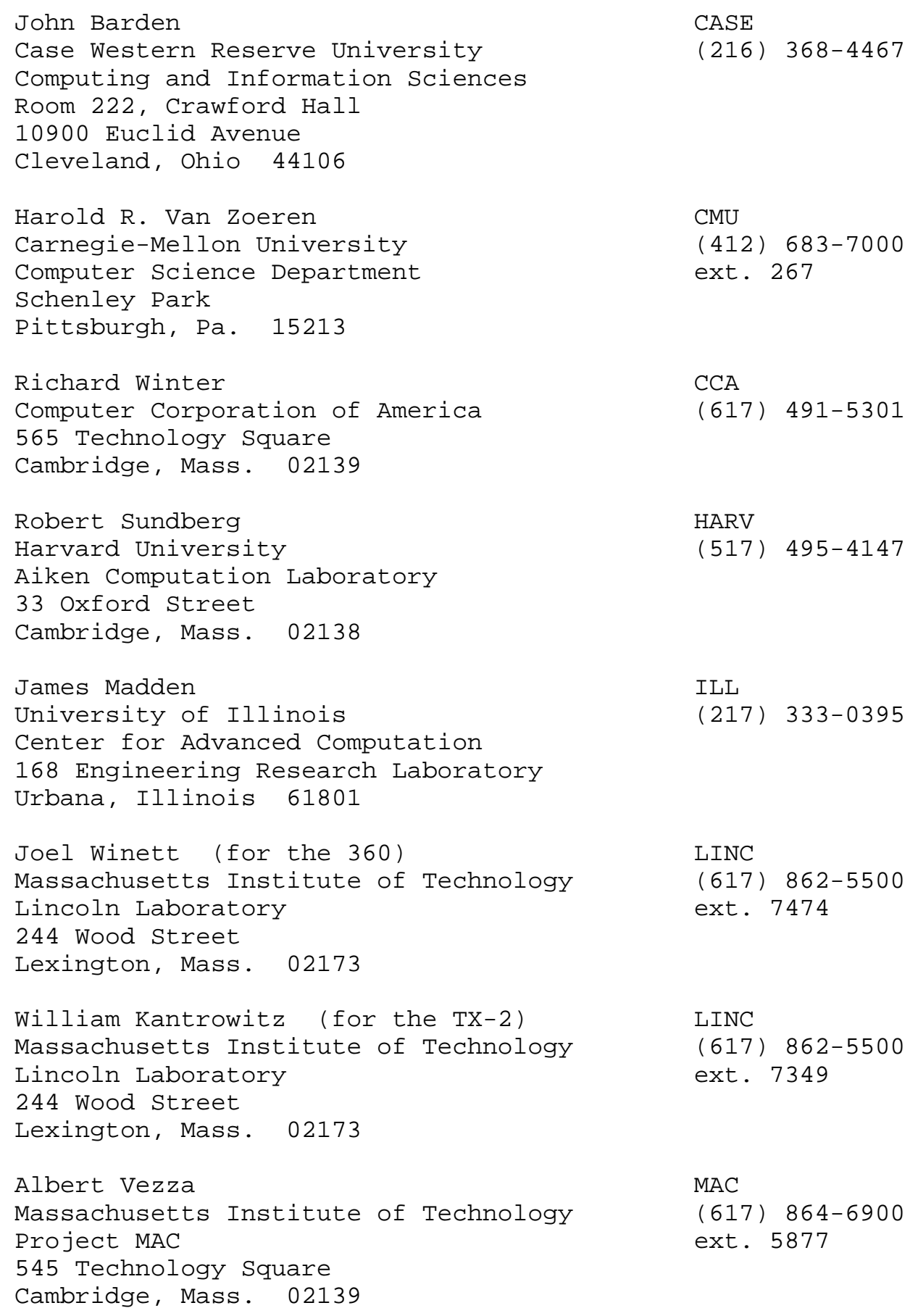

CASE

(216) 368-4467

CMU

(412) 683-7000

ext. 267

CCA

(617) 491-5301

HARV

(517) 495-4147

ILL

(217) 333-0395

ext. 7474

LINC

(617) 862-5500

ext. 7349

MAC

(617) 864-6900

ext. 5877 


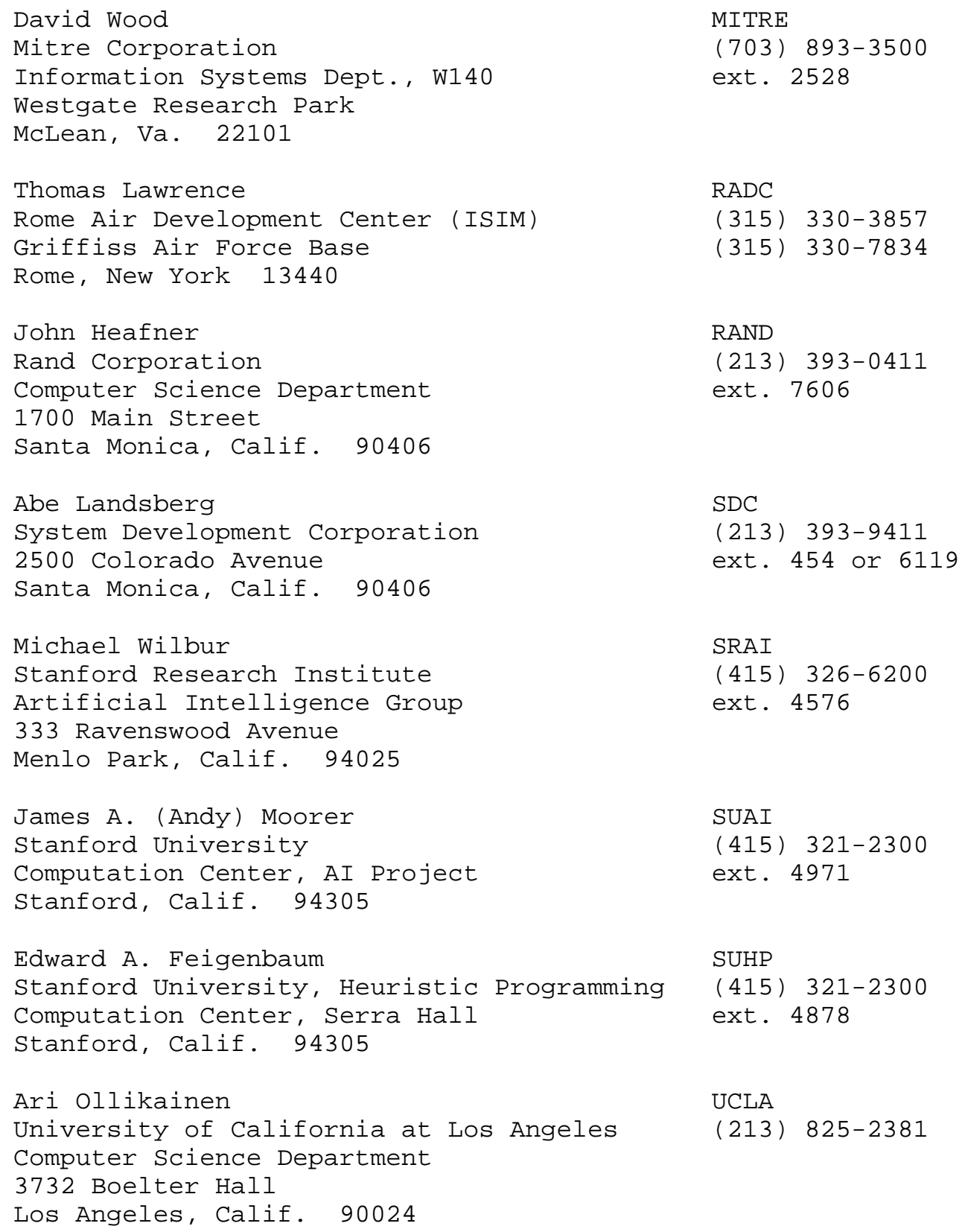

MITRE

(703) 893-3500

ext. 2528

RADC

(315) 330-3857

(315) 330-7834

RAND

(213) 393-0411

ext. 7606

$\mathrm{SDC}$

(213) 393-9411

ext. 454 or 6119

SRAI

(415) 326-6200

ext. 4576

SUAI

(415) 321-2300

ext. 4971

SUHP

(415) 321-2300

ext. 4878

UCLA

(213) 825-2381 


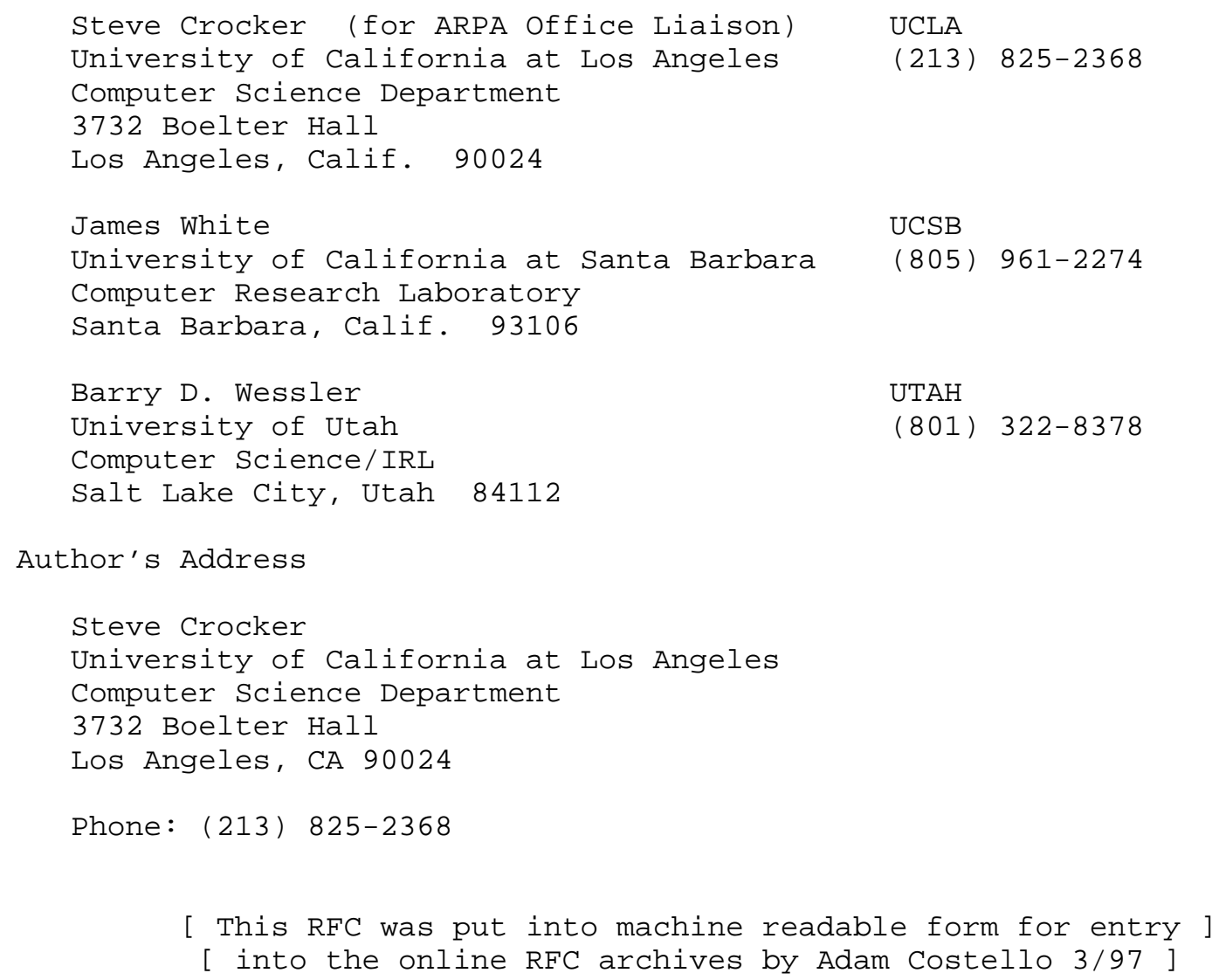

